\title{
Determination of Temperature in Solar Prominences/Filaments Using FISS Observations
}

\author{
Jongchul Chae, Hyungmin Park and Donguk Song \\ Department of Physics and Astronomy, Seoul National University, Seoul 151-742, Korea \\ email: jcchae@snu.ac.kr
}

\begin{abstract}
Using the Fast Imaging Solar Spectrograph of the 1.6 meter New Solar Telescope at Big Bear, we simultaneously took the spectral profiles of the $\mathrm{H} \alpha$ line and the Ca II line at $854.2 \mathrm{~nm}$ from prominences beyond the solar limb and filaments on the disk. The spectral data were fitted by the slab model of radiative transfer with constant source function, either with zero background intensity profile (in prominences) or with carefully constructed background intensity profile (in filaments). These observations with different perspectives and different analyses produced consistent results: temperature inside prominences/filaments ranges from 4000 to $20000 \mathrm{~K}$ with a mean of about $9500 \mathrm{~K}$. We expect that this kind of observation and analysis with higher spatial resolution and higher temporal resolution will allow us to study in detail the thermal structure and evolution of plasma in prominences.
\end{abstract}

Keywords. line: profiles, plasmas, instrumentation: spectrographs, techniques: spectroscopic, Sun: prominences

\section{Introduction}

Determining the distribution and variation of temperature inside a prominence is fundamental to understanding its thermal structure and the relevant heating/cooling processes. One can infer temperature from the profiles of the $\mathrm{H} \alpha$ line and the Ca II $854.2 \mathrm{~nm}$ line supposing these lines sense the same volume of a prominence. Since the mass of hydrogen atom much lighter than $\mathrm{Ca}$ II ion, the profile of the $\mathrm{H} \alpha$ line is more subject to thermal broadening than the Ca II line, and it is possible to determine both temperature $T$ and non-thermal speed $\xi$ from the Doppler absorption widths $\Delta \lambda_{D}$ of the two lines. Here we present the results of the determination of temperature from the simultaneously taken $\mathrm{H} \alpha$ line spectra and Ca II line spectra of limb prominences and disk filaments. The details of these works were described by Park et al. (2013) and Song et al. (2013).

\section{Observation and Analysis}

The observations were done with the Fast Imaging Solar Spectrograph (FISS) of the 1.6 meter New Solar Telescope at Big Bear Solar Observatory. The FISS is a dual-band Echelle spectrograph that can record the $\mathrm{H} \alpha$ band and the Ca II $854.2 \mathrm{~nm}$ band simultaneously (Chae et al. 2013a). The spectral range of $0.97 \mathrm{~nm}$ is covered with the sampling of $0.019 \mathrm{~nm} /$ pixel for the $\mathrm{H} \alpha$ band, and the one of $1.31 \mathrm{~nm}$, covered with $0.025 \mathrm{~nm} /$ pixel for the Ca II band. The spectral resolving power is $1.4 \times 10^{5}$ and $1.3 \times 10^{5}$, respectively. The imaging is done with the slit scan. The quality of imaging mostly depends on the performance of the adaptive optics (AO) feeding the seeing-corrected light into the 

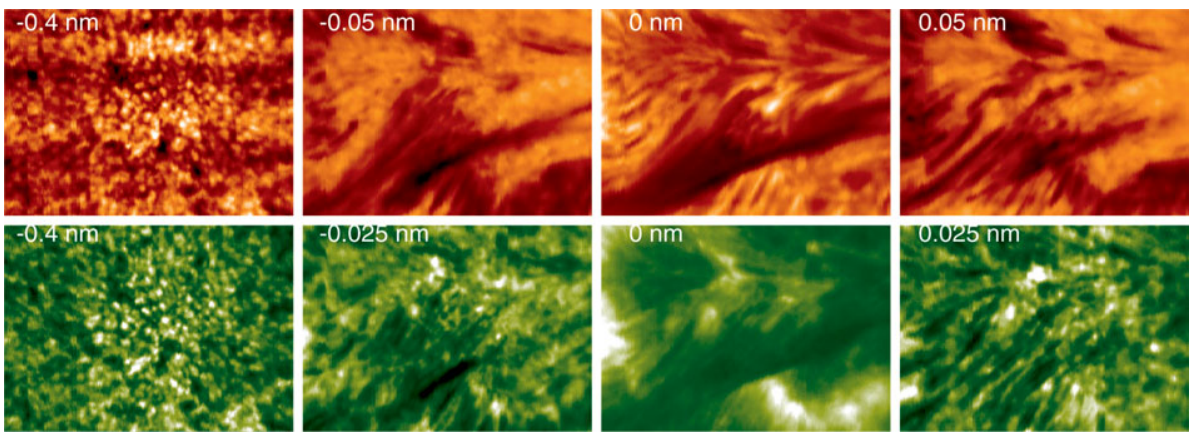

Figure 1. Raster images of a filament constructed at different wavelengths of $\mathrm{H} \alpha$ line (upper row) and the Ca II $854.3 \mathrm{~nm}$ line (lower row) from FISS observations on 2012 July 11.

instrument. The imaging quality of the FISS observations has been improved with the advance of the AO project in Big Bear. Fig. 1 illustrates the imaging quality of FISS observations done in 2012. Our results presented here came from the earlier observations done in 2010 that have poorer imaging quality. The data processing and calibration are done in the ways described by Chae et al. $(2013 \mathrm{a}, \mathrm{b})$.

The Doppler width $\Delta \lambda_{D}$ of line-emitting/absorbing plasma can be determined from the observed line profile $I_{\lambda, \text { obs }}$ by fitting it with a simple slab model of radiative transfer with source function $S$ being assumed constant over wavelength and over position along the line of sight:

$$
I_{\lambda, \mathrm{obs}}=S \cdot\left(1-\exp \left(-\tau_{\lambda}\right)\right)+I_{\lambda, \text { in }} \exp \left(-\tau_{\lambda}\right)
$$

where $\tau_{\lambda}$ is the optical thickness profile

$$
\tau_{\lambda}=\tau_{0} \exp \left(-\left[\frac{\lambda-\lambda_{0}}{\Delta \lambda_{\mathrm{D}}}\right]^{2}\right) .
$$

Note that the profile of light incident from the background $I_{\lambda, \text { in }}$ is zero in prominence observations, but is not zero and has to be estimated in filament observations. The solution in this case of non-zero background intensity is reduced to the Becker's cloud model (Beckers 1964). The background intensity profile was determined by taking average of all the profiles taken from outside the filament that have the same intensities at wavelengths away from the cores as the observed profile of interest taken from the filament.

\section{Results}

Fig. 2 shows the example of a prominence observed through the $\mathrm{H} \alpha$ line and the Ca II line. Even the prominence is optically thinner in the Ca II line than the $\mathrm{H} \alpha$ line, but the two images constructed at the cores of the two lines look very similar to each other, which supports the assumption that the two lines are emitted by the same plasma of the prominence. We find that the fittings are fairly good as illustrated in Fig. 3 . The determined Doppler widths of $0.034 \mathrm{~nm}(\mathrm{H} \alpha)$ and $0.023 \mathrm{~nm}$ (Ca II) yield the estimates: $T=10900 \mathrm{~K}$ and $\xi=7.8 \mathrm{~km} \mathrm{~s}^{-1}$. Fig. 3 also presents the scatter plot of Doppler widths 

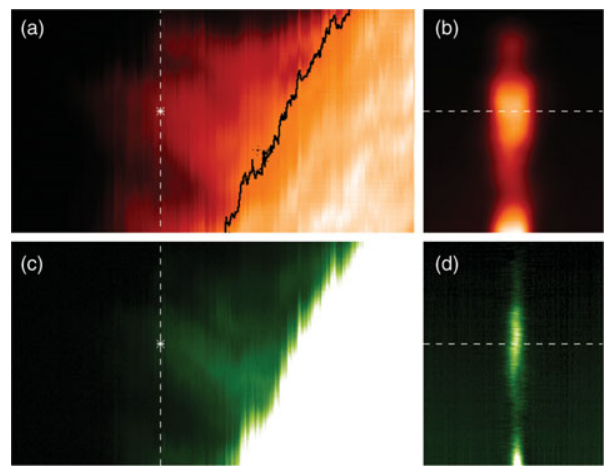

Figure 2. Left: raster images of the prominence observed on 30 June 2010 constructed at the center wavelengths of $\mathrm{H} \alpha$ line (upper) and the Ca II line (lower). Right: the spectrograms of the $\mathrm{H} \alpha$ line (upper) and the Ca II line (lower) at the slit position indicated by the dashed vertical lines.
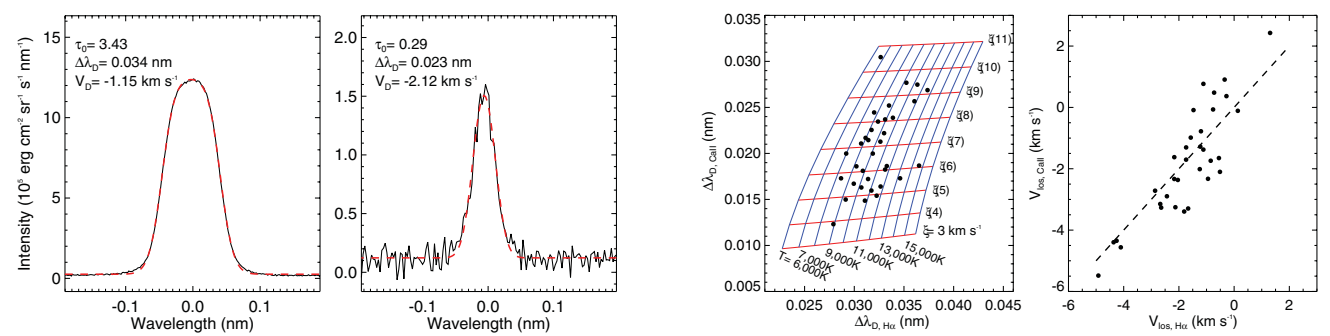

Figure 3. Left two: sesults of model fitting to the $\mathrm{H} \alpha$ line profile and the Ca II line profile. Right two: scatter plots of $\Delta \lambda_{D}(\mathrm{H} \alpha)$ vs $\Delta \lambda_{D}$ (Ca II), and $V_{\text {los }}(\mathrm{H} \alpha)$ vs $V_{\text {los }}$ (Ca II) for the prominence shown in Fig. 2. The overplotted iso- $T$ curves and the iso- $\xi$ curves allows an easy estimate of $T$ and $\xi$ from the plot.

with more number of data points. We find that $T$ here ranges from $7000 \mathrm{~K}$ to $15000 \mathrm{~K}$ with the mean of $10200 \mathrm{~K}$ and the standard deviation of $1580 \mathrm{~K}$. The right panel of Fig. 3 shows that the line-of-sight velocities determined from the two lines are close to each other, which is a support that the two lines sense the same volumes. We determined $T$ at many different parts of another prominence observed the same day, and obtained the mean of $9400 \mathrm{~K}$ and the standard deviation of $2200 \mathrm{~K}$. The third prominence was observed on a different day, 15 August 2011. The mean temperature in this prominence was $8700 \mathrm{~K}$ and the standard deviation, $1100 \mathrm{~K}$.

Fig. 4 shows the images of the filament observed on 29 July 2010. The filament is less prominent and optically thinner in the CaII line than in the $\mathrm{H} \alpha$ line, like in the limb observations. Fig. 5 illustrates the goodness of the fittings of the contrast profiles $C_{\lambda} \equiv I_{\lambda, \mathrm{obs}} / I_{\text {in }}-1$ with the cloud model. The model fitting of the profiles marked by the diamond symbol, for example, produces $\Delta \lambda_{D}$ of $0.027 \mathrm{~nm}$ for the $\mathrm{H} \alpha$ line and $0.016 \mathrm{~nm}$ for the CaII line, which can be explained by the parameter values: $T=$ $7500 \mathrm{~K}$ and $\xi=5.3 \mathrm{~km} \mathrm{~s}^{-1}$. In the same way, we obtain $T=12600 \mathrm{~K}$ and $\xi=4.4$ $\mathrm{kms}^{-1}$ for the data point marked by the circle symbol. We find that $T$ varies much with position inside the filament, with the mean of $9800 \mathrm{~K}$ and the standard deviation of $3100 \mathrm{~K}$. 

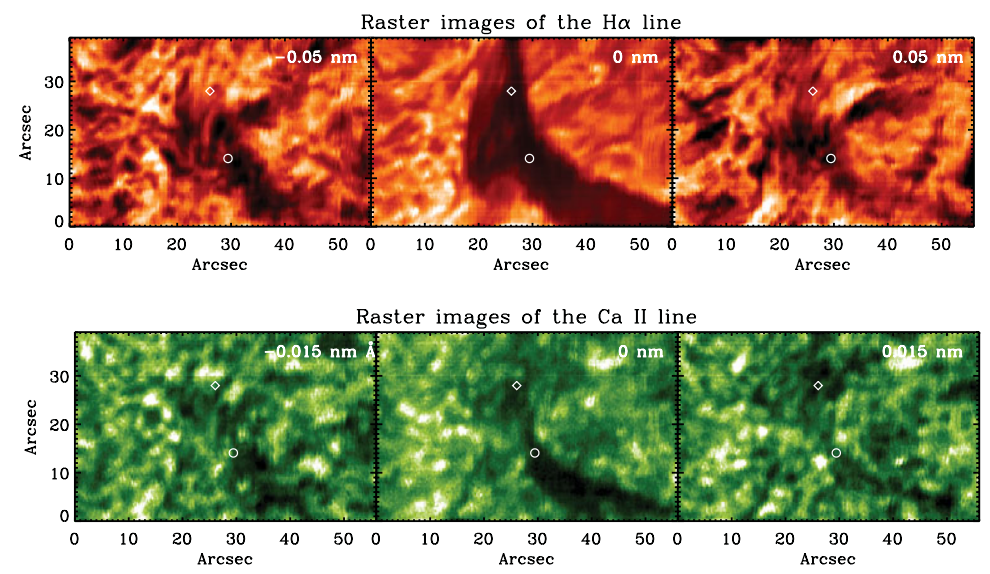

Figure 4. Raster images of the filament observed on 29 July 2010 constructed at different wavelengths of the $\mathrm{H} \alpha$ line (top) and the Ca II line (btottom).
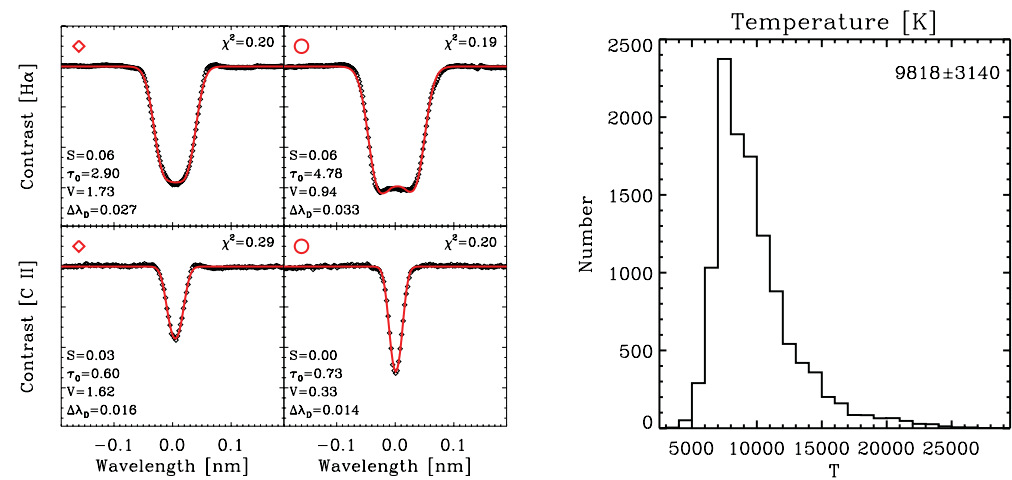

Figure 5. Left: examples of fitting of the spectra at two selected points. Right: The number distribution of temperature determined inside the filament.

\section{Conclusion}

Our limb observations of the prominences and disk observations of the filament produced consistent results on the values of temperature in prominences/filaments; the prominences/filaments have mean temperatures in the narrow range between 8700 to $10200 \mathrm{~K}$ while the variation of temperature inside each individual prominence/filament is large. The lowest temperature is around $4000 \mathrm{~K}$, and the highest temperature is $20000 \mathrm{~K}$ or higher, being in agrement with previous studies (e.g. Hirayama 1985). This consistency between limb observations and disk observations is quite encouraging to us, since the analysis of disk observations requires the determination of background intensity, while limb observations do not. The consistency indicates that our analysis of disk observations is reasonable, so that our analysis can be extended to other disk observations. It is definitely worthwhile to investigate the spatial and temporal variation of $T$ inside a prominence/filament in detail.

This work was supported by the National Research Foundation of Korea (NRF 2012R1A2A1A03670387). 


\section{References}

Beckers, J. M. 1964, A Study of the Fine Structures in the Solar Chromosphere, Ph.D. thesis, University of Utrecht

Chae, J., Park, H.-M., Ahn, K., et al. 2013a, Solar Phys., in press

Chae, J., Park, H.-M., Ahn, K., et al. 2013b, Solar Phys., in press

Hirayama, T. 1985, Solar Phys., 100, 415

Park, H., Chae, J., Song, D., et al. 2013, Solar Phys., in press

Song, D., Chae, J., Park, H., et al. 2013, Solar Phys., submitted 